\title{
A study on design methods for condenser radiator fan module mounting system
}

\section{Xiao-Ang Liu* and Xin Zhu}

School of Mechanical Engineering,

Hebei University of Technology,

Tianjin, 300130, China

Email: xiaoang314@163.com

Email: 656468301@qq.com

${ }^{*}$ Corresponding author

\section{Wenlong Shangguan}

Dongfeng Commercial Vehicle Technical Center,

Wuhan, 430056, China

Email:sgw18888@163.com

\section{Tie Wang}

School of Vehicle and Traffic, Shenyang Ligong University,

Shenyang, 110159, China

Email: wangtielucky@126.com

\section{Xiao-Li Wang}

School of Automobile and Transportation Engineering, Guangdong Polytechnic Normal University,

Guangzhou, 510665, China

Email: xlwang55@163.com

\section{Yong Chen}

School of Mechanical Engineering,

Hebei University of Technology,

Tianjin, 300130, China

Email: chenyong1585811@163.com

\begin{abstract}
Design requirements of automotive condenser radiator fan module (CRFM) mounting system are proposed. A six degrees of freedom (DOFs) CRFM model is established. The calculation method for determining the root mean square (RMS) value of mount reaction force in a CRFM mounting system under cooling fan excitation is developed. Design and calculation methods for displacement control of the CRFM under extreme load are discussed. Robustness analysis is presented through a design of experimental method. At the end of this paper, a calculation example is proposed to get the mount
\end{abstract}


stiffness and tuning point based on the design requirements for mount reaction forces and displacement of the CRFM centre of gravity. Effects of mount stiffness at linear section on natural frequencies and energy distributions of the CRFM and on RMS value of mount reaction forces are validated by a generic example.

Keywords: CRFM; condenser radiator fan module mounting system; design requirements; robustness analysis; calculation method.

Reference to this paper should be made as follows: Liu, X-A., Zhu, X., Shangguan, W., Wang, T., Wang, X-L. and Chen, Y. (2019) 'A study on design methods for condenser radiator fan module mounting system', Int. J. Heavy Vehicle Systems, Vol. 26, No. 2, pp.119-135.

Biographical notes: Xiao-Ang Liu is a Lecturer of Vehicle Engineering at Hebei University of Technology in Tianjin, China. She graduated from South China University of Technology.

Xin Zhu is a Master degree candidate of Vehicle Engineering at Hebei University of Technology in Tianjin, China.

Wenlong Shangguan is a NVH test engineer at Dongfeng Commercial Vehicle Technical Center.

Tie Wang is a Professor at Shenyang Ligong University.

Xiao-Li Wang is an Associate Professor at Guangdong Polytechnic Normal University.

Yong Chen is a Professor and Director at the New Energy Automotive Research Center in the School of Mechanical Engineering, Hebei University of Technology.

\section{Introduction}

A condenser radiator fan module (CRFM) in vehicles consists of a condenser, a radiator and a cooling fan. It is supported by some rubber mounts on the car body or subframe. These mounts and the CRFM are called CRFM mounting system. The CRFM is one of main sources of noise and vibration for the vehicle. Since the powerful engine is widely used and rotation speed and unbalance mass of the cooling fan are increased, effect of the CRFM on vehicle noise, vibration and harshness (NVH) becomes more significant.

In general load cases, the external excitation applied to the CRFM mounting system is generated by the unbalanced rotating force of the cooling fan. The excitation frequency of the CRFM is related to rotating speed of the cooling fan, which may cause resonance of the CRFM and large vibration on vehicle. In the extreme load cases, the displacement of the CRFM centre of gravity (C.G.) is relatively large and the CRFM may collide with other components. The objective of this paper is to estimate the CRFM mount stiffness under both general and extreme load cases.

In the present paper, studies on the CRFM mounting system are divided into two parts. One is concerning about the excitation of the CRFM, which is generated by cooling 
fan. Taguchi analysis method is used to find out that the primary factors contributing to vertical vibration and axial vibration are static unbalance of the fan blade and configuration of the fan's components as assembled, respectively (Morgan, 1997). The parameters which affect the noise generated by fans are analysed by Wu et al. (1997, 1998).

The other is on design methods for CRFM mounting system. A linear model for CRFM mounting system is established to calculate the vibration of CRFM C.G., series of experiments are taken to compare different isolator groups under excitation of chassis and cooling fan vibration (Yang et al., 2012, 2013). However, there are few researches on the design requirements, analysis methods and non-linear characteristic of the CRFM mounting system. From the published papers, design methods for other different mounting systems are studied, which can be references for CRFM mounting system. In the powertrain mounting system (PMS), mount stiffness at linear section in three directions of its local coordinate system (LCS), mount locations and orientations can be obtained by optimisation of the natural frequencies, energy distributions of the powertrain rigid mode (Liu and Shangguan, 2014; Shangguan, 2009; Yu et al., 2001; Shangguan and Chen, 2012; El Hafidi et al., 2010). Mount stiffness at non-linear sections and its tuning points in the force vs. displacement curve are determined by powertrain motion control strategies (Shangguan and Hou, 2006). The effect of mount stiffness and location variations on modal characteristics of the mounting systems are studied in Qatu et al. (2002), Sirafi et al. (2006), Sirafi et al. (2006) and Iqbal and Qatu (2003). Through a design of experiment (DOE) approach, the mounts and directions which play major role in the improvement of NVH are identified.

In this paper, the design requirements for the CRFM mounting system are proposed firstly. Based on six degrees of freedom (DOFs) CRFM model and the derived excitation forces, design philosophies and calculation methods for the CRFM mounting system are proposed. The mount stiffness at linear and non-linear section and the tuning points in three orthogonal coordinate axes can be obtained, respectively. Thirdly, a DOE approach is adopted and effects of mount stiffness on natural frequencies and energy distributions of the CRFM and on RMS value of mount reaction forces are analysed. The design results are validated by a generic example.

\section{Design requirements for the CRFM mounting system}

During the design of the CRFM mounting system, three significant parts should be taken into consideration:

1 Natural frequencies of CRFM and CRFM mount reaction forces: To reduce the vibration transmitted from the CRFM to the car body through the CRFM mounts, mount reaction forces should be optimised. The peak frequency of the mount reaction force is affected by the natural frequency of CRFM.

2 Displacement control for CRFM C.G.: To avoid the collision of CRFM with other components, displacement of CRFM C.G. under extreme load cases should be controlled. Therefore, the non-linear relation of force and displacement of the CRFM mount should be optimised. 
3 Robustness analysis for the design result: In industry, the design parameters of the CRFM mounting system are usually the mount stiffness and locations. Variations of design parameters on CRFM mounting system should be analysed to verify the robustness of design results.

1 Design requirements for CRFM mount reaction forces and natural frequencies

In order to reduce the vibration transmitted from CRFM to the car body through mounting system, mount reaction forces should be decreased. The design requirement for mount reaction force in different frequency ranges is shown in Figure 1. The peak frequency is at $25-28 \mathrm{~Hz}$, which is corresponding to the second order of the engine excitation frequency at idle. $f_{1}$ is the hop/tramp frequency of the unsprung mass. $f_{3}$ is the natural frequency of the steering wheel. According to the temperature of the radiator, there are two work modes for the cooling fan: low rotation speed mode and high rotation speed mode. $f_{2}$ is the excitation frequency of CRFM when the engine is on and air conditioner (AC) is on at idle, $f_{4}$ is the excitation frequency of CRFM when the engine is off and the $\mathrm{AC}$ is on. To avoid resonance, mount reaction forces in these four frequency ranges are designed relatively small.

The design philosophy of natural frequencies for the CRFM rigid mode can be divided into two different categories. One is to set the natural frequencies of the CRFM far away from the idle frequency of the engine and the hop/tramp frequency of the unsprung mass. Then, the resonance of the CRFM can be efficiently avoided. The other design philosophy is to use the CRFM as a mass damper to absorb the idle vibration of the engine. In this paper, the CRFM is designed as a mass damper, natural frequency of CRFM in Z-direction is designed at $25-28 \mathrm{~Hz}$, which is the same as the peak frequency of mount reaction force requirement curve. All the six natural frequencies of the CRFM should be lower than $f_{4} / \sqrt{2}=35.4 \mathrm{~Hz}$ and should not be the same as $f_{1}, f_{2}, f_{3}, f_{4}$.

Through the design requirement for mount reaction forces and natural frequencies, mount stiffness at linear section can be designed.

\section{Motion control requirements for the CRFM}

To control the displacement of CRFM C.G. within the limits under different extreme load cases, such as maximum acceleration in $X$-, $Y$ - and $Z$-direction, motion control requirements should be satisfied. The maximum accelerations acting on the CRFM and displacement limitations for the CRFM C.G. in each load case are listed in Table 1.

Table 1 Load cases description and displacement limitations for C.G. of CRFM

\begin{tabular}{llcc}
\hline Case no. & Load case description & $\begin{array}{c}\text { g'load on CRFM } \\
\left(1 \mathrm{~g}=9.8 \mathrm{~m} / \mathrm{s}^{2}\right)\end{array}$ & $\begin{array}{c}\text { Requirement } \\
(\mathrm{mm})\end{array}$ \\
\hline 1 & Maximum acceleration in positive $X$-direction & $+7.1 \mathrm{~g}$ & +5 \\
2 & Maximum acceleration in negative $X$-direction & $-7.1 \mathrm{~g}$ & -5 \\
3 & Maximum acceleration in positive $Y$-direction & $+5.9 \mathrm{~g}$ & +3 \\
4 & Maximum acceleration in negative $Y$-direction & $-5.9 \mathrm{~g}$ & -3 \\
5 & Maximum acceleration in positive Z-direction & $+11.7 \mathrm{~g}$ & +4.5 \\
6 & Maximum acceleration in negative Z-direction & $-11.7 \mathrm{~g}$ & -4.5 \\
\hline
\end{tabular}


Figure 1 Design requirement for RMS value of mount dynamic reaction force (see online version for colours)

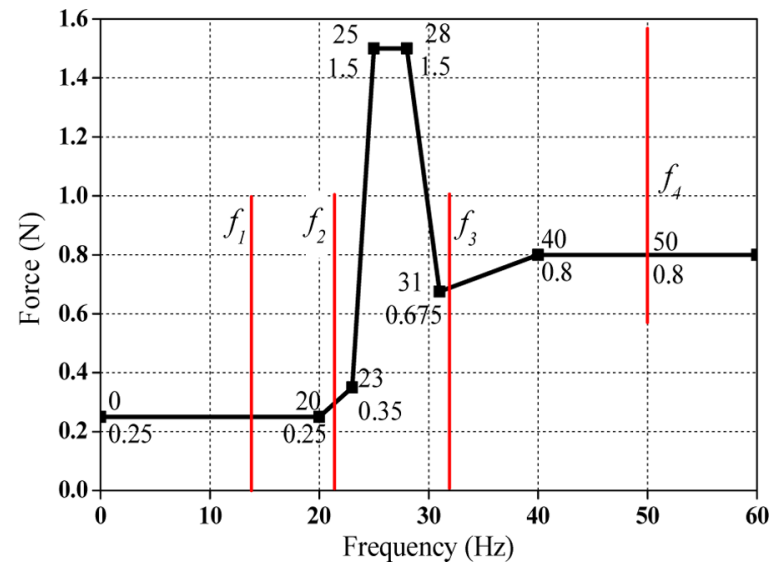

\section{Design, calculation and robustness analysis methods for the CRFM mounting system}

\subsection{Calculation method for RMS value of mount dynamic reaction forces}

Figure 2 shows the six DOFs model of a CRFM mounting system. In this model, the CRFM is treated as a rigid body which has six DOFs. It is supported on the car body or subframe by four mounts, which are the left-upper (LU), right-upper (RU), left-low (LL) and right-low (RL) mount.

Figure 2 The model of a CRFM mounting system (see online version for colours)

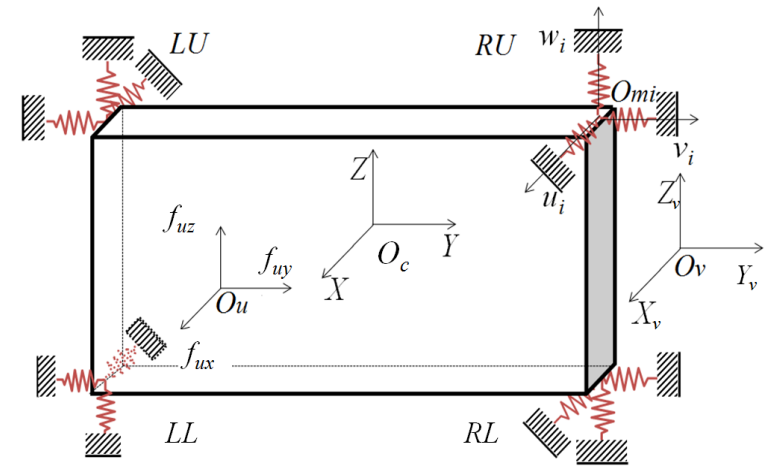

The right-hand side CRFM coordinate system and the mount LCS are denoted as $O_{c}-X Y Z$ and $O_{m i}-u_{i} v_{i} w_{i}(i=1,2,3,4)$, respectively. The origins of $O_{c}-X Y Z$ and $O_{m i}-u_{i} v_{i} w_{i}$ are located at C.G. of the CRFM and the centre of each mount, respectively. Each axis of $O_{c}-X Y Z$ are parallel to the axes in vehicle coordinate system (VCS) $O_{v}-X_{v} Y_{v} Z_{v}$. The positive $X$-axis points to rear of the vehicle. The $Z$-axis direction is normal to the horizontal plane and the positive is upward, the $Y$-axis follows the righthand rule. 
The displacement of C.G. of the CRFM is defined as $\mathbf{X}=\left(x, y, z, \theta_{x}, \theta_{y}, \theta_{z}\right)^{T}$, where $x, y, z$ are the translation displacements and $\theta_{x}, \theta_{y}, \theta_{z}$ are the rotation displacements.

When the fan rotates, a centrifugal force will be generated by the eccentric mass on point $O_{u}$ in Figure 2. The fan rotates around the axis of motor, which is parallel to the $X$-axis of CRFM coordinate system. Decompose the centrifugal force along the $Y$ - and $Z$ axis, the excitation force of CRFM mounting system are defined as

$$
f_{u}=\left\{\begin{array}{c}
0 \\
m_{e} r_{e} \omega^{2} \cos (\omega t) \\
m_{e} r_{e} \omega^{2} \sin (\omega t)
\end{array}\right\},
$$

where $m_{e}$ is the eccentric mass, $r_{e}$ is the eccentric arm, $\omega$ is the angular velocity of the cooling fan, $t$ is time.

Transmit the unbalance force from the application point $O_{u}$ to the origin $O_{c}$ of $O_{c}-X Y Z$, the external excitation force $F_{e}$ applied to the CRFM in $O_{c}-X Y Z$ is expressed as

$$
F_{e}=\left\{\frac{f_{u}}{\tilde{r}_{u} f_{u}}\right\},
$$

where $\tilde{r}_{u}$ is the skew-symmetric matrix (Sirafi et al., 2006) of the position vector $\vec{r}_{u}=x_{u} \vec{i}+y_{u} \vec{j}+z_{u} \vec{k}, \quad x_{u}, y_{u}$ and $z_{u}$ are the coordinate of application point $O_{u}$ of the force $f_{u}$ in $O_{c}-X Y Z$.

Under the external force $F_{e}$, displacement of the mount $i$ in $\operatorname{LCS} O_{m i}-u_{i} v_{i} w_{i}$ is expressed as

$$
d_{i}=\left[\begin{array}{l:l}
I & -\tilde{r}_{i}
\end{array}\right] X,
$$

where $\tilde{r}_{i}$ is the skew-symmetric matrix of positive vector $r_{i}=\left(x_{i}, y_{i}, z_{i}\right)^{T}, x_{i}, y_{i}$ and $z_{i}$ are the coordinate of mount $i$ in $O_{c}-X Y Z$.

If the mount stiffness is linear, then the force at mount $i$ in LCS $O_{m i}-u_{i} v_{i} w_{i}$ is expressed as

$$
f_{i}=k_{i} d_{i}
$$

where $k_{i}$ is the complex stiffness matrix (Shangguan, 2009) of mount $i$ in its LCS $O_{m i}-u_{i} v_{i} w_{i}$.

Then reaction forces and moments generated by mount $i$ applied to C.G. of CRFM in $O_{c}-X Y Z$ are expressed as

$$
\begin{aligned}
& f_{m i}=-A_{i} k_{i} A_{i}^{T} d_{i}=\left[\begin{array}{l:l}
-A_{i} k_{i} A_{i}^{T} & A_{i} k_{i} A_{i}^{T} \tilde{r}_{i}
\end{array}\right] X,
\end{aligned}
$$

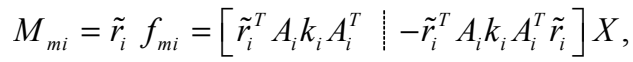


where $A_{i}$ is the transformation matrix from the CRFM coordinate system to the LCS of mount $i$. The transformation matrix can be easily formed from the orientation of a mount with respect to the CRFM coordinate system, which can be defined using Euler angles, direction cosines, etc. (Swanson et al., 1993).

Then, the motion equation of the CRFM under four mount reaction force and external force is expressed as

$$
M \ddot{X}=\sum_{i=1}^{4}\left\{\frac{f_{m i}}{M_{m i}}\right\}+F_{e},
$$

where $M$ is the mass matrix, which is the same as PMS mass matrix (El Hafidi et al., 2010).

Substituting equations (2), (5), (6) into equation (7), one gets

$$
M \ddot{X}+\left[\begin{array}{c:c}
\sum_{i=1}^{n} A_{i} k_{i} A_{i}^{T} & -\sum_{i=1}^{n} A_{i} k_{i} A_{i}^{T} \tilde{r}_{i} \\
\hdashline-\sum_{i=1}^{n} \tilde{r}_{i}^{T} A_{i} k_{i} A_{i}^{T} & \sum_{i=1}^{n} \tilde{r}_{i}^{T} A_{i} k_{i} A_{i}^{T} \tilde{r}_{i}
\end{array}\right] X=\left\{\begin{array}{c}
f_{u} \\
\hdashline \tilde{r}_{u} f_{u}
\end{array}\right\} .
$$

Then, the displacement of CRFM C.G. in the frequency domain can be calculated. The reaction force of mount $i$ in CRFM coordinate system $O_{c}-X Y Z$ can be calculated through equation (5). Maximal boundary curve of the transmitted force of each mount is specified in RMS value, the RMS value of the mount reaction force is calculated by

$$
f_{r m s i}=\sqrt{\left(f_{m i x}^{2}+f_{m i y}^{2}+f_{m i z}^{2}\right) / 3}
$$

where $f_{\text {mix }}, f_{\text {miy }}, f_{\text {miz }}$ are the reaction forces of mount $i$ in the $X$-, $Y$ - and $Z$-direction of $O_{c}-X Y Z$, respectively.

Through the calculation method above, the design results of mount stiffness at linear section are obtained through satisfying the design requirement for RMS value of mount dynamic reaction force in Figure 1.

\subsection{Design method for displacement control of a CRFM}

Linear mount characteristics are not sufficient because of package constraints and progressive mount characteristics have to be designed. The relation of force and displacement of a mount in each directions of its LCS is simplified as piecewise linear as shown in Figure 3. It is seen that there are five ranges. The linear stiffness $\left(K_{3}\right)$ is obtained by satisfying the design requirements for mount reaction force of the CRFM. The non-linear stiffness $\left(K_{1}, K_{2}, K_{4}, K_{5}\right)$ and the coordinates of tuning points $\left(P_{1}, P_{2}, P_{3}, P_{4}\right)$ of each mount in Figure 3 are tuned based on the CRFM motion control requirements. Maximum feasible displacement happens under maximum acceleration load case. Then, the displacement limitations in Table 1 should locate at $P_{0}-P_{1}$ or $P_{4}-P_{5}$ range. 
Figure 3 Force vs. displacement relation of a mount in one direction of its LCS (see online version for colours)

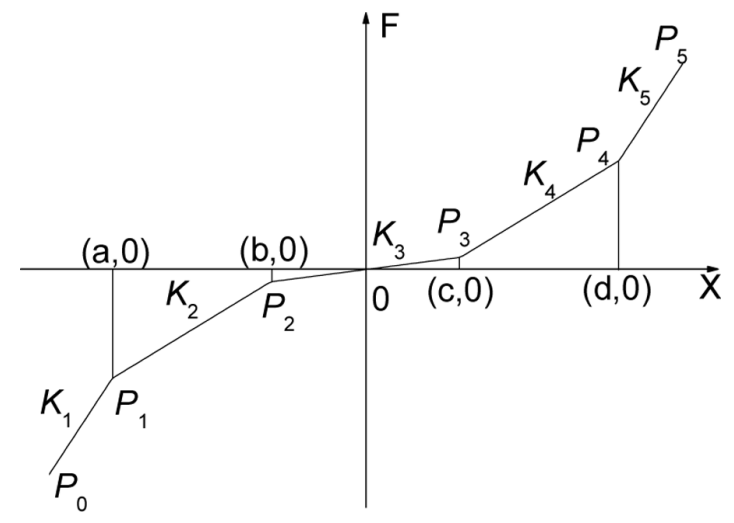

If the mount stiffness is non-linear, the force at mount $i$ under the excitation force is not the same as equation (4) and is expressed as

$$
f_{i}=k_{i} d_{i}+\Delta_{i}
$$

where $\boldsymbol{\Delta}_{i}$ are the correction term of mount $i$.

The values of stiffness $k_{i}$ and correction term $\Delta_{i}$ are listed in Table 2. It is seen that their values are different at different ranges of the displacement.

Table 2 Values of stiffness and correction terms

\begin{tabular}{llc}
\hline$x$ range & $k_{i}$ & $\Delta$ \\
\hline$x \leq a$ & $K_{1}$ & $-K_{1} a+K_{2}(a-b)+K_{3} b$ \\
$a<x \leq b$ & $K_{2}$ & $-K_{2} b+K_{3} b$ \\
$b<x<c$ & $K_{3}$ & 0 \\
$c \leq x<d$ & $K_{4}$ & $-K_{4} c+K_{3} c$ \\
$x \geq d$ & $K_{5}$ & $-K_{5} d+K_{4}(d-c)+K_{3} c$ \\
\hline
\end{tabular}

Then reaction forces and moments generated by mount $i$ applied to C.G. of CRFM in $O_{c}-X Y Z$ are expressed as

$$
\begin{aligned}
& f_{m i}=-A_{i} k_{i} A_{i}^{T} d_{i}-A_{i} \Delta_{i} \\
& M_{m i}=\tilde{r}_{i} f_{m i} .
\end{aligned}
$$

Then, the equation of the CRFM under four mount reaction force and quasi-static external load is expressed as 


$$
\begin{aligned}
& \sum_{i=1}^{4}\left\{\begin{array}{c}
\left\{f_{m i}\right. \\
\left.\hdashline M_{m i}\right\}+F_{q e}=0, \quad K X=F_{q e}-\Delta, \\
K=\left[\begin{array}{c:c}
\sum_{i=1}^{n} A_{i} k_{i} A_{i}^{T} & -\sum_{i=1}^{n} A_{i} k_{i} A_{i}^{T} \tilde{r}_{i} \\
\hdashline-\sum_{i=1}^{n} \tilde{r}_{i}^{T} A_{i} k_{i} A_{i}^{T} & \sum_{i=1}^{n} \tilde{r}_{i}^{T} A_{i} k_{i} A_{i}^{T} \tilde{r}_{i}
\end{array}\right], \quad \Delta=\left[\begin{array}{c}
\sum_{i=1}^{n} A_{i} \Delta_{i} \\
\hdashline \sum_{i=1}^{n} \tilde{r}_{i} A_{i} \Delta_{i}
\end{array}\right]
\end{array}\right.
\end{aligned}
$$

where $F_{q e}$ is the quasi-static load applied on the CRFM C.G.

An iterative algorithm should be used to get the optimised relation of force vs. displacement of the CRFM mount in each direction of the LCS through equations (11)-(14). The steps are described as follows:

Step 1: Assuming the stiffness of each mount is at linear section, then the displacements of CRFM C.G. and each mount under extreme load cases can be obtained;

Step 2: Check the displacements of each mount obtained in step 1 to see if they are in the linear range. If yes, output the displacements of each mount and then stop. If no, record the ranges that the displacements for each mount in its LCS located, and then go to next step.

Step 3: Constitute the new matrices of $k_{i}$ and $\Delta_{i}$ with the range data in step 2 and the formulae in Table 2, then form the stiffness matrix $K$ and correction matrix $\Delta$, respectively.

Step 4: Calculate the displacements of CRFM C.G., then the displacements of each mount in its LCS can be obtained.

Step 5: Check the displacements of each mount in its LCS obtained in step 4 to see in which ranges the displacements are located, and record the ranges of the displacements for each mount in its LCS. If the ranges are the same as in step 2, output the calculated results and then stop, otherwise, go to step 3 .

\subsection{Robustness analysis method for a CRFM mounting system}

In robustness analysis, the purpose is to study the effect of variability in CRFM mount stiffness on natural frequencies and energy distributions of CRFM and on RMS value of mount reaction forces. The rubber material used in these mounts when installed in the vehicle could have as much as $\pm 15 \%$ stiffness variability. This variability could be from batch to batch variation in material or assembly variation. So the stiffness of the upper mount and lower mount are assumed to vary independently by $\pm 15 \%$ and there are no variations in other parameters of the CRFM mounting system. This leads to 12 parameters to vary. However, the stiffness of two upper mounts and two lower mounts are the same, respectively, the number of independent variables is reduced to 6 . Then a two level, eight runs DOE (Table 3) is constructed to perform the analysis. In Table 3, ' 1 ' and ' 2 ' mean the value of $85 \%$ and $115 \%$ design mount stiffness, respectively. $k_{u u}, k_{u v}$, $k_{u w}$ represent the stiffness of upper mount in $u$-, $v$ - and $w$-direction in the LCS, respectively. $k_{l u}, k_{l v}, k_{l w}$ represent the stiffness of lower mount in $u$-, $v$ - and $w$ direction in the LCS, respectively. 
Table 3 A design of experiment matrix with eight runs and two levels for six variables for mount stiffness $(\mathrm{N} / \mathrm{mm})$ variation

\begin{tabular}{lcccccc}
\hline Run no. & $k_{u u}$ & $k_{u v}$ & $k_{u w}$ & $k_{l u}$ & $k_{l v}$ & $k_{l w}$ \\
\hline 1 & 1 & 1 & 1 & 1 & 1 & 1 \\
2 & 1 & 1 & 1 & 2 & 2 & 2 \\
3 & 1 & 2 & 2 & 1 & 1 & 2 \\
4 & 1 & 2 & 2 & 2 & 2 & 1 \\
5 & 2 & 1 & 2 & 1 & 2 & 1 \\
6 & 2 & 1 & 2 & 2 & 1 & 2 \\
7 & 2 & 2 & 1 & 1 & 2 & 2 \\
8 & 2 & 2 & 1 & 2 & 1 & 1 \\
\hline
\end{tabular}

\section{Examples}

\subsection{Input parameters}

The mass of CRFM is $10 \mathrm{~kg}$. The moments and products of inertia of CRFM in CRFM coordinate system $O_{c}-X Y Z$ are listed in Table 4 . The eccentric mass $m_{e}$ on cooling fan is $80 \mathrm{~g}$, the eccentric arm $r_{e}$ is $1 \mathrm{~mm}$. The coordinates of CRFM C.G., application point $O_{\mathrm{u}}$ of the unbalanced force and locations of each mount in the VCS are listed in Table 5. The $u_{i}-, v_{i}$ - and $w_{i}$-axes of the LCS $O_{m i}-u_{i} v_{i} w_{i}$ of each mount are parallel to the $X-, Y$ - and $Z$-axis of CRFM coordinate system $O_{c}-X Y Z$, respectively. Since the dynamic stiffness at linear section and loss angle of rubber mount are hardness-dependent in low frequency $(0-20 \mathrm{~Hz})$, define the dynamic-to-static ratio is 1.3 and the loss angle is 14 degree. The original static stiffness of each mount in $u_{i}-, v_{i}$ - and $w_{i}$-axis at linear section are 20,20 and $50 \mathrm{~N} / \mathrm{mm}$, respectively.

\subsection{Design results}

The design parameters of the CRFM mounting system are stiffness of each mount. Considering the low-cost, interchangeability and light-weight characteristics, the LU and RU mount are designed the same and the LL and RL mount are designed the same. Take the fatigue into consideration, the stiffness in $w$-axis should be larger than those in $u$ - and $v$-axes. To satisfy the requirements in Figure 1, natural frequencies of CRFM in the $Z$-direction should locate at $25-28 \mathrm{~Hz}$. The design stiffnesses of each mount are listed in Table 6.

Table 4 The moments and products of inertia of CRFM in $O_{c}-X Y Z\left(\mathrm{~kg} \cdot \mathrm{m}^{2}\right)$

\begin{tabular}{lccccc}
\hline$I_{x x}$ & $I_{y y}$ & $I_{z z}$ & $I_{x y}$ & $I_{y z}$ & $I_{x z}$ \\
\hline 0.511 & 0.155 & 0.405 & 0.021 & -0.006 & -0.001 \\
\hline
\end{tabular}


Table 5 Coordinates of CRFM C.G., application point of unbalance force and locations of each mount in VCS (mm)

\begin{tabular}{lccc}
\hline Object & $X$ & $Y$ & \multicolumn{1}{c}{$Z$} \\
\hline C.G. of CRFM & -636.32 & -22.91 & 240.45 \\
Application point of unbalance force & -608.03 & -59.90 & 242.73 \\
RU mount & -658.01 & 362.50 & 474.53 \\
LU mount & -659.12 & -364.70 & 474.53 \\
RL mount & -658.01 & 362.50 & 22.53 \\
LL mount & -659.12 & -364.70 & 22.53 \\
\hline
\end{tabular}

Table 6 The design result of static mount stiffness at linear section in its LCS $\left(\mathrm{N} \cdot \mathrm{mm}^{-1}\right)$

\begin{tabular}{lccc}
\hline Mount & $u$ & $V$ & $W$ \\
\hline LU \& RU & 23 & 17 & 54 \\
LL \& RL & 29 & 21 & 52 \\
\hline
\end{tabular}

The six natural frequencies of the CRFM rigid mode are listed in Table 7. From the table, the original natural frequencies in $X$ - and $Y$-direction are nearly the same, and the frequency in $Z$-direction is below the requirement. The original energy distributions in $X$ - and $Y$-direction are quite low, which means the vibrations in $X$ - and $Y$-directions are coupled. Through the design stiffness, five natural frequencies are smaller than the main excitation frequency of cooling fan (corresponding to $f_{4}$ in Figure 1, which is about $50 \mathrm{~Hz}$ ) multiply $1 / \sqrt{2}$, except for the Rx-direction. It is because the natural frequencies distributions of the CRFM are large, requirements for lower than $50 \mathrm{~Hz} / \sqrt{2}=35.4 \mathrm{~Hz}$ cannot be satisfied simultaneously, however, the interval of natural frequency in Rx-direction and the excitation frequency are larger than $2 \mathrm{~Hz}$. The natural frequency in $Z$-direction is $25.8 \mathrm{~Hz}$ and satisfies the design requirement for the dynamic vibration absorber $(25-28 \mathrm{~Hz})$. The interval of CRFM natural frequencies and natural frequencies of each direction are also satisfied. All the energy distributions in each direction are above $75 \%$, which are much better than the original ones.

Table 7 The natural frequencies and energy distributions of the CRFM

\begin{tabular}{llcccccc}
\hline & & $X$ & $Y$ & $Z$ & $R x$ & $R y$ & $R z$ \\
\hline Natural frequency/Hz & Original & 16.1 & 16.2 & 24.7 & 44.7 & 30.6 & 29.4 \\
& Design results & 18.4 & 15.8 & 25.8 & 45.8 & 34.3 & 33.5 \\
Energy distribution/\% $\%$ & Original & 56.3 & 56.6 & 85.4 & 98.6 & 77.2 & 90.3 \\
& Design results & 99.3 & 99.9 & 93.9 & 97.7 & 79.0 & 85.5 \\
\hline
\end{tabular}

The original and design results of frequency response characteristics of RMS value of mount reaction forces are shown in Figure 4(a) and (b). From Figure 4(a), the mount reaction forces in $20-25 \mathrm{~Hz}$ are larger, this is because the original natural frequency in $Z$-direction is lower than the requirement. After design, the mount reaction forces are 
reduced. The peaks of the frequency response characteristic curves are located at about $26 \mathrm{~Hz}$, which is corresponding to the natural frequency of the CRFM in Z-direction. Since the CRFM is treated as a dynamic vibration absorber, vibrations generated from the engine are absorbed by the CRFM.

In Figure 4(b), there is also a peak at $16 \mathrm{~Hz}$, which is corresponding to the natural frequency of the CRFM in $Y$-direction. Considering the supporting and displacement control functions of each mount, the stiffness of them in each axis should not be too small. As a result, the RMS value of mount reaction force may be larger than the requirements in some frequency range, such as at $15-20 \mathrm{~Hz}$. However, the RMS values of mount reaction forces of each mount are still quite small. In conclusion, the designs of mount stiffness at linear section satisfy the design requirements.

According to the design requirements for displacement control of CRFM C.G. in different load cases in Table 1, mount stiffness at non-linear sections and coordinates of tuning points can be designed using the calculation and design methods in Section 3.3. The design results of mount stiffness in the LCS are listed in Table 8.

The displacements of CRFM C.G. under each extreme load cases are listed in Table 9. It is seen that the design of mount stiffness at non-linear section in Table 8 satisfies the design requirements. From Table 9, the displacements of CRFM C.G. in the excitation direction (in bold) are increased obviously when the mount stiffness is linear, which may cause a collision between the CRFM and other components. The displacement of CRFM mounts will be also increased if the mount stiffness is linear, which may decrease the fatigue lift of mounts. As a result, it is of great importance that the design for the relation of force vs. displacement of each mount is non-linear in quasi-static loads.

Figure 4 Target and RMS values of mount reaction forces: (a) original and (b) design results (see online version for colours)

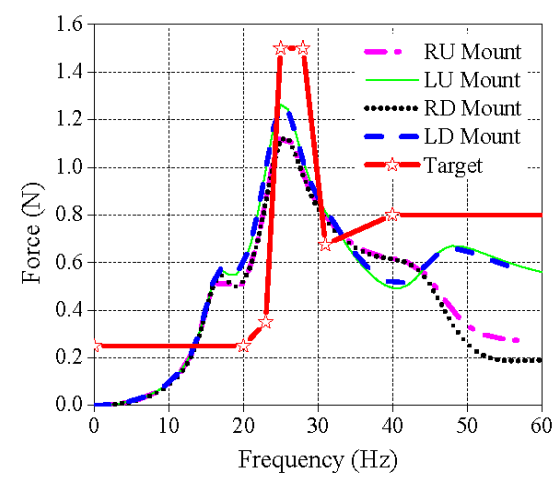

(a)

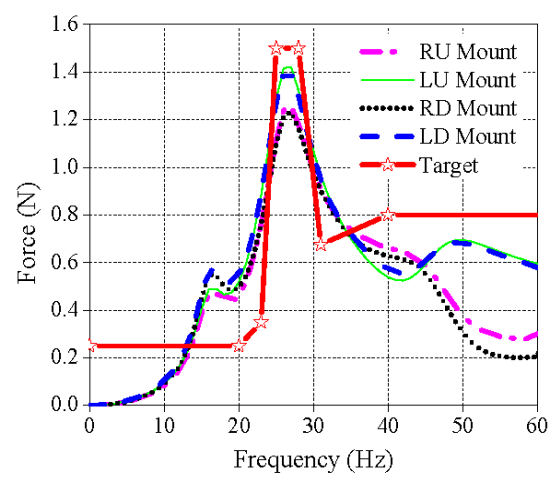

(b)

The relation of force vs. displacement for the RU and LL mount in three directions of the LCS is shown in Figure 5. According to the displacement of CRFM C.G., the displacement of the RU and LL mounts in six extreme load cases are marked in the figures. It is seen that the displacements of the mount are at non-linear section under each extreme load case.

The reaction forces of each CRFM mount are calculated and listed in Table 10 when the mount stiffness is linear or non-linear. From Table 10, some reaction forces in bold 
are increased when the mount stiffness is non-linear. It is because the stiffness at the non-linear section is about 4-21 times larger than stiffness at linear section. However, the increases of reaction forces are not large.

Table 8 Static stiffness and $x$ coordinates of the tuning points for each mount

\begin{tabular}{|c|c|c|c|c|c|c|c|c|c|c|}
\hline \multirow[b]{2}{*}{ Mount } & \multirow{2}{*}{$\begin{array}{c}\text { LCS of a } \\
\text { mount }\end{array}$} & \multicolumn{5}{|c|}{ Stiffness for piecewise linear $(\mathrm{N} / \mathrm{mm})$} & \multicolumn{4}{|c|}{$\begin{array}{c}x \text {-coordinate of tuning } \\
\text { point } / \mathrm{mm}\end{array}$} \\
\hline & & $K_{1}$ & $K_{2}$ & $K_{3}$ & $K_{4}$ & $K_{5}$ & $P_{1}$ & $P_{2}$ & $P_{3}$ & $P_{4}$ \\
\hline \multirow[t]{3}{*}{ LU \& RU } & $\mathrm{u}$ & 200 & 50 & 23 & 50 & 200 & -4.5 & -2.5 & 2.5 & 4.5 \\
\hline & $\mathrm{v}$ & 255 & 45 & 17 & 45 & 255 & -2.5 & -1.5 & 1.5 & 2.5 \\
\hline & $\mathrm{w}$ & 230 & 108 & 54 & 80 & 180 & -3.5 & -2 & 1 & 2.5 \\
\hline \multirow[t]{3}{*}{ LL \& RL } & $\mathrm{u}$ & 210 & 70 & 29 & 70 & 210 & -4 & -3 & 3 & 4 \\
\hline & $\mathrm{v}$ & 450 & 50 & 21 & 50 & 450 & -2.5 & -1.5 & 1.5 & 2.5 \\
\hline & w & 220 & 104 & 52 & 80 & 180 & -3 & -2 & 1 & 3.5 \\
\hline
\end{tabular}

Table 9 Design requirements and calculation results of displacement of CRFM C.G. if the mount stiffness is linear or non-linear

\begin{tabular}{llrrrr}
\hline & & \multicolumn{3}{c}{ Displacement of CRFM C.G. } & \\
\cline { 3 - 4 } Case no. & Mount stiffness & $X / m m$ & $Y / m m$ & Z/mm & Requirement $(\mathrm{mm})$ \\
\hline \multirow{2}{*}{1} & Linear & $\mathbf{6 . 9 0}$ & 0.03 & -0.05 & +5 \\
& Nonlinear & 4.34 & 0.00 & -0.01 & \\
2 & Linear & $-\mathbf{6 . 9 0}$ & -0.02 & 0.05 & -5 \\
& Nonlinear & -4.35 & 0.00 & 0.03 & \\
3 & Linear & 0.02 & $\mathbf{7 . 7 9}$ & 0.01 & +3 \\
& Nonlinear & 0.02 & 2.73 & 0.00 & \\
4 & Linear & -0.02 & $-\mathbf{7 . 7 9}$ & -0.01 & -3 \\
& Nonlinear & -0.02 & -2.73 & 0.00 & +4.5 \\
5 & Linear & -0.09 & 0.01 & $\mathbf{5 . 6 5}$ & \\
& Nonlinear & -0.09 & 0.00 & 3.88 & -4.5 \\
6 & Linear & 0.09 & -0.01 & $-\mathbf{5 . 6 5}$ & \\
& Nonlinear & 0.09 & 0.00 & -3.25 & \\
& & & & &
\end{tabular}

Table 10 Reaction forces $(N)$ of each CRFM mount if the mount stiffness is linear or nonlinear

\begin{tabular}{llcccc}
\hline Case no. & Mount stiffness & LU mount & RU mount & LL mount & RL mount \\
\hline \multirow{2}{*}{ Linear } & 98.43 & 109.82 & 97.89 & 111.61 \\
& Nonlinear & 97.90 & $\mathbf{1 1 0 . 3 6}$ & $\mathbf{9 8 . 4 5}$ & 111.05 \\
3 & Linear & 82.58 & 81.62 & 93.75 & 93.56 \\
& Nonlinear & $\mathbf{8 3 . 9 1}$ & $\mathbf{8 3 . 5 9}$ & 92.08 & 91.63 \\
5 & Linear & 137.26 & 151.92 & 157.71 & 175.10 \\
& Nonlinear & 131.93 & 143.82 & $\mathbf{1 6 1 . 5 3}$ & $\mathbf{1 8 4 . 6 9}$ \\
\hline
\end{tabular}


Figure 5 The relation of force vs. displacement for RU and LL mount and their displacements under extreme load cases in three axes of LCS: (a) RU mount and (b) LL mount (see online version for colours)

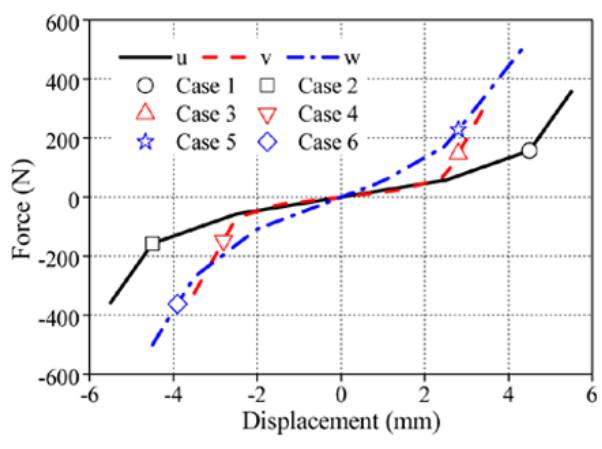

(a)

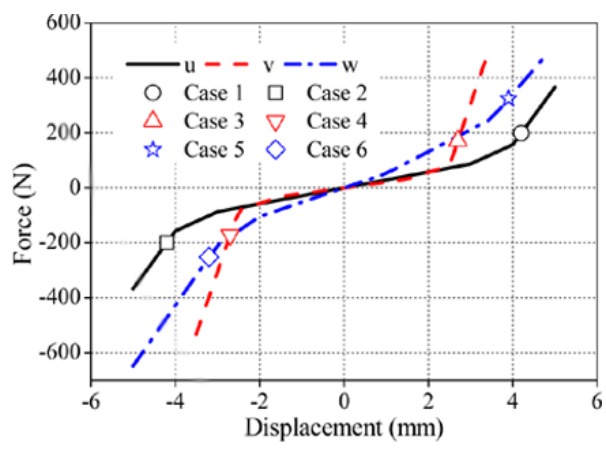

(b)

\subsection{Robustness analysis}

According to the mount stiffness in Table 6, stiffnesses of upper and lower mounts in two levels are listed in Table 11. Eight runs are conducted and calculation results are shown in Tables 12, 13 and Figure 6.

In Table 12 and 13, the Normal lines are calculated by stiffness in Table 6 . Natural frequencies and energy distributions underlined are those whose variations are above 5\% comparing to the Normal line. Natural frequencies in bold and italic in Table 12 cannot satisfy the requirements in Section 4.2. Energy distributions in bold and italic in Table 13 are better than the results in Normal line. From Tables 12 and 13, No. 6 and 8 cases are the worst cases considering natural frequencies and energy distributions of the CRFM. In No. 1 case, variations of each mount stiffness are the same, then energy distributions do not change, however, the variation of natural frequencies equals the square root of variation of mount stiffness.

Table 11 Mount stiffness (N/mm) in LCS in two levels

\begin{tabular}{lcccccc}
\hline Level & $k_{u u}$ & $k_{u v}$ & $k_{u w}$ & $k_{l u}$ & $k_{l v}$ & $k_{l w}$ \\
\hline $1(-15 \%)$ & 19.6 & 14.5 & 45.9 & 24.7 & 17.9 & 44.2 \\
$2(+15 \%)$ & 26.5 & 19.6 & 62.1 & 33.4 & 24.2 & 59.8 \\
\hline
\end{tabular}

In Figure 6, peaks of mount reaction forces in No. 1 and 8 are out of $25-28 \mathrm{~Hz}$ which can verified from natural frequency in $Z$-direction listed in Table 12. Average mount reaction forces in No. 3 and 6 at $30-40 \mathrm{~Hz}$ are larger than design requirements. Tighter control of the mount stiffness is recommended as installed in the vehicle. This includes searching for better methods to measure such stiffness when the mounts are installed in the vehicle pre-loaded with assembly variations. 
Table 12 Robustness of natural frequencies of the CRFM to variations in mount stiffness

\begin{tabular}{lcccccc}
\hline Run no. & $X$ & $Y$ & $Z$ & $R x$ & $R y$ & $R z$ \\
\hline 1 & $\underline{16.9}$ & $\underline{14.6}$ & $\underline{\mathbf{2 3 . 8}}$ & $\underline{42.2}$ & $\underline{31.6}$ & $\underline{30.9}$ \\
2 & 17.9 & 15.9 & 25.8 & 45.8 & 34.5 & 33.9 \\
3 & $\underline{16.9}$ & 15.7 & 26.9 & $\underline{48.6}$ & $\underline{32.5}$ & $\underline{30.9}$ \\
4 & 17.9 & $\underline{16.9}$ & 25.9 & 46.2 & 34.5 & 33.9 \\
5 & 18.2 & 15.9 & 25.8 & 45.9 & 34.3 & 33.1 \\
6 & $\underline{19.7}$ & 14.6 & $\underline{27.6}$ & $\underline{48.3}$ & $\mathbf{3 6 . 7}$ & $\underline{35.9}$ \\
7 & 18.2 & $\underline{16.9}$ & 25.7 & 46.1 & 34.3 & 33.1 \\
8 & $\underline{19.7}$ & $\underline{15.7}$ & $\underline{\mathbf{2 4 . 0}}$ & $\underline{42.8}$ & $\underline{\mathbf{3 6 . 3}}$ & $\underline{\mathbf{3 5 . 8}}$ \\
Normal & $\mathbf{1 8 . 4}$ & $\mathbf{1 5 . 8}$ & $\mathbf{2 5 . 8}$ & $\mathbf{4 5 . 8}$ & $\mathbf{3 4 . 3}$ & $\mathbf{3 3 . 5}$ \\
Max & $\mathbf{1 9 . 7}$ & $\mathbf{1 6 . 9}$ & $\mathbf{2 7 . 6}$ & $\mathbf{4 8 . 6}$ & $\mathbf{3 6 . 7}$ & $\mathbf{3 5 . 9}$ \\
Min & $\mathbf{1 6 . 9}$ & $\mathbf{1 4 . 6}$ & $\mathbf{2 3 . 8}$ & $\mathbf{4 2 . 2}$ & $\mathbf{3 1 . 6}$ & $\mathbf{3 0 . 9}$ \\
Average & $\mathbf{1 8 . 2}$ & $\mathbf{1 5 . 8}$ & $\mathbf{2 5 . 7}$ & $\mathbf{4 5 . 7}$ & $\mathbf{3 4 . 3}$ & $\mathbf{3 3 . 4}$ \\
\hline
\end{tabular}

Figure 6 Robustness of mount reaction forces to variations in mount stiffness (see online version for colours)

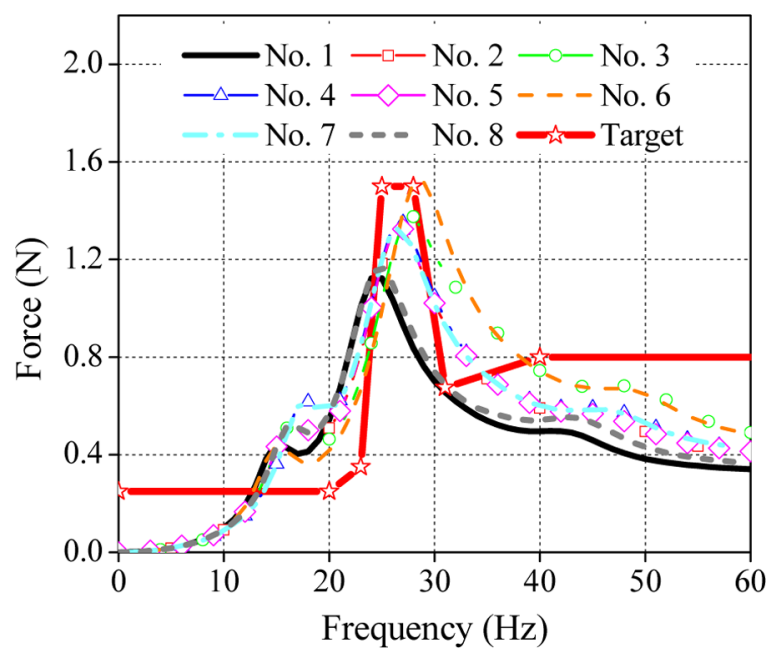

Table 13 Robustness of energy distributions of the CRFM mounting system to variations in mount stiffness

\begin{tabular}{lcccccc}
\hline Run no. & $X$ & $Y$ & $Z$ & $R x$ & $R y$ & $R z$ \\
\hline 1 & 99.3 & 99.9 & 93.9 & 97.7 & 79.0 & 85.5 \\
2 & 96.3 & 99.8 & 94.4 & 97.5 & $\underline{\mathbf{8 9 . 4}}$ & $\underline{\mathbf{9 8 . 6}}$ \\
3 & 99.2 & 99.8 & $\underline{76.9}$ & 98.7 & $\underline{\mathbf{7 4 . 2}}$ & $\underline{\mathbf{9 6 . 9}}$ \\
4 & 96.2 & 99.7 & 94.2 & 97.7 & $\underline{\mathbf{8 9 . 2}}$ & $\underline{\mathbf{9 8 . 3}}$ \\
5 & 99.4 & 99.8 & 93.6 & 97.6 & $\underline{\mathbf{7 4 . 7}}$ & 81.2 \\
\hline
\end{tabular}


Table 13 Robustness of energy distributions of the CRFM mounting system to variations in mount stiffness (continued)

\begin{tabular}{lcccccc}
\hline Run no. & $X$ & $Y$ & $Z$ & $R x$ & $R y$ & $R z$ \\
\hline 6 & 99.3 & 99.9 & 93.9 & 97.4 & 78.9 & 85.7 \\
7 & 99.4 & 99.7 & 93.7 & 97.8 & $\underline{74.2}$ & $\underline{80.4}$ \\
8 & 99.2 & 99.9 & 97.9 & 93.6 & $\underline{52.0}$ & $\underline{\mathbf{5 6 . 5}}$ \\
Normal & $\mathbf{9 9 . 3}$ & $\mathbf{9 9 . 9}$ & $\mathbf{9 3 . 9}$ & $\mathbf{9 7 . 7}$ & $\mathbf{7 9 . 0}$ & $\mathbf{8 5 . 5}$ \\
Max & $\mathbf{9 9 . 4}$ & $\mathbf{9 9 . 9}$ & $\mathbf{9 7 . 9}$ & $\mathbf{9 8 . 7}$ & $\mathbf{8 9 . 4}$ & $\mathbf{9 8 . 6}$ \\
Min & $\mathbf{9 6 . 2}$ & $\mathbf{9 9 . 7}$ & $\mathbf{7 6 . 9}$ & $\mathbf{9 3 . 6}$ & $\mathbf{5 2 . 0}$ & $\mathbf{5 6 . 5}$ \\
Average & $\mathbf{9 8 . 6}$ & $\mathbf{9 9 . 8}$ & $\mathbf{9 2 . 5}$ & $\mathbf{9 7 . 3}$ & $\mathbf{7 6 . 7}$ & $\mathbf{8 5 . 4}$ \\
\hline
\end{tabular}

\section{Conclusions}

- The six DOFs CRFM mounting system model is established based on the design requirements. Under the unbalanced excitation force generated by eccentric mass of the cooling fan, equations to calculate the displacement of CRFM C.G. and mount reaction forces are derived. The design and calculation methods for displacement control of the CRFM are proposed. The design philosophies of mount stiffness at linear and non-linear sections are presented. The design results are validated by an example.

- Robustness of the CRFM mounting system with respect to the mount stiffness is studied. Effects of mount stiffness on six natural frequencies and energy distributions of the CRFM and on RMS value of mount reaction forces are analysed. Tighter control of the mount stiffness is recommended as installed in the vehicle.

\section{Acknowledgement}

This work was supported by the Natural Science Foundation of China (Grant No. 51705128 and No. 51505091).

\section{References}

El Hafidi, A., Martin, B., Loredo, A. and Jego, E. (2010) 'Vibration reduction on city buses: determination of optimal position of engine mounts', Mechanical Systems and Signal Processing, Vol. 24, No. 7, pp.2198-2209.

Iqbal, J. and Qatu, M. (2003) Robustness of Axle Mount System for Driveline NVH, SAE Technical Paper Series, 2003-01-1485.

Liu, X-A. and Shangguan, W-B. (2014) 'Elastomeric components for noise and vibration isolation and control in the automotive industry', Encyclopedia of Automotive Engineering. John Wiley $\&$ Sons, Ltd., New York.

Morgan, W. (1997) Vibration Analysis of an Electric Motor Fan Assembly, SAE Technical Paper Series, 971854. 
Qatu, M., Sirafi, M. and Johns, F. (2002) 'Robustness of powertrain mount system for noise, vibration and harshness at idle', Proceedings of the Institution of Mechanical Engineers, Part D: Journal of Automobile Engineering, Vol. 216, No. 10, pp.805-810.

Shangguan, W. (2009) 'Engine mounts and powertrain mounting systems: a review', International Journal of Vehicle Design, Vol. 49, No. 4, pp.237-258.

Shangguan, W. and Hou, Z. (2006) 'Strategies and calculation methods for automotive powertrain motion control under quasi-static loads', Proceedings of the Institution of Mechanical Engineers, Part D: Journal of Automobile Engineering, Vol. 220, No. 8, pp.1131-1138.

Shangguan, W.B. and Chen, D.M. (2012) 'A method for calculating the displacements of a powertrain and mounts in a powertrain mounting system including torque struts under quasistatic loads', Proceedings of the Institution of Mechanical Engineers, Part D: Journal of Automobile Engineering, Vol. 226, No. 5, pp.634-647.

Sirafi, M., Chang, Y. and Qatu, M.S. (2006) 'Robustness of mount systems for idle NVH, Part I: centre of gravity (CG) mounts', International Journal of Vehicle Noise and Vibration, Vol. 2, No. 4, pp.317-333.

Sirafi, M., Chang, Y. and Qatu, M.S. (2006) 'Robustness of mount systems for idle NVH, Part II: pendulum mounts', International Journal of Vehicle Noise and Vibration, Vol. 2, No. 4, pp.334-340.

Swanson, D.A., Wu, H.T. and Ashrafiuon, H. (1993) 'Optimization of aircraft engine suspension systems', Journal of Aircraft, Vol. 30, No. 6, pp.979-984.

Wu, S.F., Su, S. and Shah, H. (1998) 'Noise radiation from engine cooling fans', Journal of Sound and Vibration, Vol. 216, No. 1, pp.107-132.

$\mathrm{Wu}$, S.F., Su, S.G. and Shah, H.S. (1997) 'Modelling of the noise spectra of axial flow fans in a free field', Journal of Sound and Vibration, Vol. 200, No. 4, pp.379-399.

Yang, J., Hao, Z-Y., Ge R-W. and Zheng, X. (2013) 'Optimization of engine cooling module vibration based on a genetic algorithm', Journal of Vibration and Shock, Vol. 32, No. 1, pp.164-168.

Yang, J., Hao, Z-Y., Ge, R-W. and Zheng, K. (2012) 'Optimization of engine cooling module vibration', Journal of Zhejiang University (Engineering Science), Vol. 46, No. 12, pp.2194-2200.

Yu, Y., Naganathan N.G. and Dukkipati R.V. (2001) 'A literature review of automotive vehicle engine mounting systems', Mechanism and Machine Theory, Vol. 36, No. 1, pp.123-142. 